Anaesthesist 2010 - 59:289-292

DOI 10.1007/s00101-010-1705-y

Online publiziert: 26. März 2010

(c) Springer-Verlag 2010
T. Standl

Klinik für Anästhesie, Operative Intensiv- und Palliativmedizin, Städtisches Klinikum

Solingen, Akademisches Lehrkrankenhaus der Universität Köln, Solingen

\title{
Glaube oder Leben?
}

\section{Schwierige Entscheidungsfindung im perioperativen Management bei Zeugen Jehovas}

Die Glaubensgemeinschaft der Zeugen Jehovas wurde im ausgehenden 19. Jh. in den USA von Charles Russell gegründet und versteht sich als kirchlich organisierte Religionsgemeinschaft, die sich durch eine besonders starke Missionstätigkeit hervorhebt. Weltweit zählt sie nach eigenen Angaben etwa 7,1 Mio. Anhänger, die in über 100.000 sog. Versammlungen (Bezeichnung für ortsansässige Gemeinden) organisiert sind. In Deutschland leben etwa 165.000 Zeugen Jehovas, in Österreich und in der Schweiz etwa 20.000 resp. 18.0oo. Die Zeugen Jehovas leiten ihre Glaubensgrundsätze direkt und sehr wörtlich aus der Bibel ab. Diese enthält demnach die von Gott geoffenbarte Wahrheit und bildet damit Grundlage der gesamten Lehre. Die für die Glaubensgemeinschaft charakteristische Ablehnung der Transfusion von Blut und Blutbestandteilen bezieht sich deshalb auch auf Bibelzitate, in denen Blut eine besonders religiöse Bedeutung zugesprochen wird und worin bereits den Israeliten die Verwendung von But ausschließlich für sakrale Handlungen erlaubt gewesen ist.

\section{(7) Blut wird eine besonders religiöse Bedeutung zugesprochen}

Die Verweigerung der Transfusion von Blut und Blutbestandteilen, schlimmstenfalls auch von vital indizierten Bluttransfusionen, stellt die behandelnden Ärzte und hier insbesondere die Anästhesisten im klinischen Alltag vor eine beson- dere Herausforderung. Nicht zuletzt wegen möglicher forensischer Folgen muss sich der Anästhesist grundsätzlich und vor der Behandlung eines Patienten der Glaubensgemeinschaft der Zeugen Jehovas mit den rechtlichen Hintergründen und der damit verbundenen Aufklärungs- sowie Dokumentationspflicht, einschließlich der daraus möglicherweise resultierenden Haftungsgrundlagen, auseinandersetzen [1]. Von ebenso großer Bedeutung ist es, eine Strategie für die perioperative Behandlung dieser Patienten zu haben. Die rechtlichen Aspekte des perioperativen Managements bei Ablehnung von Fremdblut durch Zeugen Jehovas werden in der vorliegenden Ausgabe Der Anaesthesist von Prof. Dr. Ulsenheimer ausführlich und klar dargestellt [2]. Aus diesem Beitrag geht hervor, dass bei erwachsenen Patienten der Glaubensgemeinschaft der Zeugen Jehovas vor elektiven Eingriffen im Fall einer Ablehnung ein intensives und genauestens dokumentiertes Aufklärungsgespräch, am besten mit Zeugen, stattfinden muss. In diesem Aufklärungsgespräch müssen die möglicherweise auch lebensbedrohlichen Konsequenzen der Ablehnung einer Transfusion von Blut und Blutprodukten klar angesprochen sowie deren Ablehnung vom Patienten durch seine Unterschrift bestätigt werden. Etwas anders ist die Bewertung bei Patienten, die sich in einem lebensbedrohlichen Zustand befinden oder aufgrund z. B. einer Intensivbehandlung nicht ansprechbar und geschäftsfähig sind. Hier kann es selbst beim Vorhan- densein einer entsprechenden Patientenverfügung, in der die Verweigerung einer Transfusion von Blutprodukten schriftlich dargestellt ist, im begründetem Einzelfall gerechtfertigt sein, unter Berücksichtigung standes- und berufsrechtlicher Gründe eines Arztes (hippokratischer Eid) das Leben des Patienten zu schützen und eine Bluttransfusion durchzuführen. So ist nach einem Urteil des Oberlandesgerichts München aus dem Jahr 2002 der Arzt nicht in jedem Fall gehalten, der eindeutigen Patientenverfügung einer Nichttransfusion zu folgen, auch wenn diese eine Freizeichnungsklausel für ihn enthält [3]. Somit ergibt sich eine eingeschränkte Verbindlichkeit auch einer in der Patientenverfügung dargelegten eindeutigen Willensäußerung gegen Fremdbluttransfusionen in bestimmten Fällen. In den entsprechenden Fachzeitschriften nahezu aller klinischen Fächer hat es in den letzten Jahren aufgrund dieser grundsätzlich eindeutigen, im Einzelfall jedoch bisweilen schwierigen Rechtslage verschiedene Übersichtsartikel gegeben $[1,4,5,6]$.

Erwähnenswert im Beitrag von K. Ulsenheimer ist die Feststellung, dass selbst im Fall der Betreuung eines nichtentscheidungsfähigen Patienten und vorliegender Patientenverfügung vom behandelnden Arzt das Betreuungsgericht eingeschaltet werden kann, das dann durch die Einsetzung eines vorläufigen Betreuers eine Entscheidung zugunsten einer Transfusion von Blut oder Blutbestandteilen möglich machen kann [2]. Dies ist auch im Zusammenhang mit der perioperativen Betreu- 
Infobox 1 Zusatz zum Behandlungsvertrag

Hiermit setze ich Sie davon in Kenntnis, dass ich bei meiner medizinischen Behandlung der Verabreichung von Bluttransfusionen (von Vollblut, roten Blutkörperchen, weißen Blutkörperchen, Blutplättchen oder Blutplasma) nicht zustimme und in dieser Hinsicht meinen Behandlungsauftrag ausdrücklich limitiere. Ich stimme der Verabreichung von blutlosen Expandern und der Anwendung anderer blutloser Behandlungsmethoden zu.

Ich treffe diese medizinisch-religiöse Entscheidung als ein Zeuge Jehovas.

Mir ist bewusst, dass die behandelnden Ärzte möglicherweise eine Transfusion von Blut oder Blutbestandteilen für notwendig erachten, um mein Leben zu bewahren oder die Rekonvaleszenz zu fördern. Ich teile diese Ansicht jedoch nicht und halte an der hier gegebenen Erklärung unter allen Umständen fest.

Ich habe mir meine Entscheidung sorgfältig überlegt und treffe diese Entscheidung im Rahmen des Selbstbestimmungsrechts als Patient. Meine „Willenserklärung“ wird nicht dadurch unwirksam, dass ich bewusstlos oder handlungsunfähig bin.

Ich befreie die behandelnden Ärzte, das Krankenhaus und das Krankenhauspersonal insoweit von der Haftung für jegliche Schäden, die bei kunstgerechter Versorgung auf meine Ablehnung von Bluttransfusionen zurückgeführt werden könnten. Dieser Wille ist auch für meine Erben bindend.

Ort, Datum

Name

Unterschrift

ung von Minderjährigen, die aufgrund ihres Entwicklungsstatus noch keine natürliche Einsichtsfähigkeit haben, von weitreichender Bedeutung. Falls es die Zeit erlaubt, wird in diesen Fällen über das Jugendamt oder direkt beim Familiengericht einer anderen Person als den Eltern das Sorgerecht für die Heilbehandlung übertragen, sodass eine rechtswirksame Transfusion durchgeführt werden kann. In Notfällen wie z. B. nach Verkehrsunfällen wird sich der zuständige Arzt mit hoher Wahrscheinlichkeit für die Erhaltung von Leben und Gesundheit des Kindes sowie eine Applikation von Blut und Blutbestandteilen entscheiden.

Immer mehr Kliniken und deren Abteilungen sehen sich mit dem Wunsch der Mitglieder des sog. Verbindungskomitees der Zeugen Jehovas konfrontiert, Verträge über die Behandlung von Zeugen Jehovas abzuschließen und sich darin einerseits zur Behandlung von Patienten aus der Glaubensgemeinschaft der Zeugen Jehovas zu verpflichten, andererseits aber den Wunsch nach Nichttransfusion mit allen möglichen Konsequenzen zu akzeptieren. Für einwilligungsfähige Patienten vor Elektiveingriffen haben die Verbindungskomitees Zusatzformulare zum Behandlungsvertrag entworfen (• Infobox 1).

\section{( Eine Strategie zur Vermeidung von anämisch bedingter Hypoxie und Koagulopathie muss vorgehalten werden}

Im Fall der perioperativen Behandlung eines Zeugen Jehovas (unabhängig, ob auf der Basis abgeschlossener Verträge oder nicht) muss vor elektiven Eingriffen bei erwachsenen, geschäftsfähigen Patienten immer eine Strategie zur möglichen Vermeidung der anämisch bedingten Hypoxie [7] und der schweren Koagulopathie vorgehalten werden. Diesem Thema ist der ausführliche Leitartikel von Habler u. Voß [8] gewidmet. Es ist das Verdienst der Autoren, diese klinisch relevante und wichtige Fragestellung aus anästhesiologischer Sicht bearbeitet zu haben. Hier kommen ausführlich die möglichen sowie von den Zeugen Jehovas akzeptierten Vermeidungsstrategien und alternativen Behandlungsmethoden zur Transfusion von Fremdblut und Blutbestandteilen zur Darstellung. Da die Eigenblutspende von Zeugen Jehovas strikt abgelehnt wird, bleiben neben der permissiven Anämie insbesondere operationsvorbereitende Maßnahmen wie die Eisensubstitution, die Applikation von Erythropoetin oder ggf. bei gefäßreichen Tumoren die radiologisch interventionelle Embolisation [9]. Da alle Formen der kristalloiden und künstlichen kolloidalen Lösungen akzeptiert werden, bietet sich intraoperativ eine isovolämische Hämodilution an; die früher propagierte hypervolämische Hämodilution sollte aufgrund von ungünstigen kardiozirkulatorischen Wirkungen und Effekten auf das Endothel und die Glykokalyx [10] nicht mehr durchgeführt werden. Anästhesiologisch kommen die kontrollierte Hypotension bei Nichtrisikopatienten und bestimmte medikamentöse, die Blutgerinnung unterstützende Therapien infrage [11]. Bei Operationen, die sich nicht in septischen und tumorösen Gebiet befinden, kommt unter der Bedingung, dass eine Zwischenlagerung der gewonnenen Erythrozytenkonzentrate vermieden wird, die maschinelle Autotransfusion als fakultativ akzeptiertes Verfahren hinzu. Ebenso unterliegt die Akzeptanz von Gerinnungsfaktoren und Humanalbumin als Kolloid der individuellen Einzelentscheidung.

Große Hoffnungen haben in den letzten 10 bis 15 Jahren künstliche Sauerstoffträger wie die Perfluorocarbone (PFC) und die chemisch aufbereiteten modifizierten Hämoglobinlösungen geweckt [12]. Perfluorocarbone konnten eindrucksvoll zeigen, dass sie eine hervorragende mehrstündige „Bridging“-Funktion unter der Bedingung einer Beatmung mit 100\%igem Sauerstoff im Rahmen einer augmentierten Hämodilution übernehmen können [13]. Nachteil dieser rein chemisch basierten Lösungen, die Sauerstoff physikalisch lösen, ist neben der benötigten hohen inspiratorischen Sauerstoffkonzentration $\left(\mathrm{F}_{\mathrm{I}} \mathrm{O}_{2} \mathrm{O}, 5-1, \mathrm{O}\right)$ die zeitliche Limitierung des Effekts von mehreren Stunden, sodass nur eine überschaubare Überbrückung des Sauerstoffträgerverlusts realisiert werden kann. Bedauerlicherweise haben die PFC-Lösungen (die von den Zeugen Jehovas akzeptiert werden, da sie keinerlei organische oder blutähnliche Bestandteile haben) keine Zulassung erhalten und stehen für die klinische Behandlung nicht zur Verfügung.

Anders verhält sich dies bei den Derivaten aus menschlichen oder tierischen Erythrozyten, die „hemoglobin-based oxygen carriers" (HBOC), unter denen seit 2001 Hemopure $^{\circledR}$ in Südafrika zur Behandlung der perioperativen Anämie bei Erwachsenen zugelassen ist $[14,15]$. Für HBOC gibt es zahlreiche klinische und tierexperimentelle Studien, die ein wesentlich längeres Bridging als bei PFC belegen, z. B. im Rahmen kardiochirurgischer Operationen [16], einer perioperativen Hämodilution [17] oder in lebensgefährlichen anämischen Situationen [18]. Auch Kasuistiken bei Zeugen Jehovas belegen, dass aufgrund des Einsatzes dieser Lösungen Patienten mit ex- 
trem niedrigen Ausgangshämoglobinwerten überlebt haben $[19,20,21]$. Obwohl alle HBOC-Lösungen vasokonstriktorische Eigenschaften aufgrund des „Scavenging“-Effekts von Stickstoffmonoxid (NO) haben, konnten tierexperimentelle Studien und Kasuistiken zeigen, dass die Gewebeoxygenierung poststenotisch oder im Rahmen von Reperfusionsmodellen durch HBOC (aber auch durch PFC) signifikant verbessert werden kann [22, 23, 24, 25, 26]. Bei HBOC wird es den Glaubensangehörigen der Zeugen Jehovas freigestellt, ob sie eine perioperative Applikation akzeptieren. Bedauerlicherweise ist Hemopure ${ }^{\varpi}$ nur im Einzelfall der therapeutischen ärztlichen Entscheidung auf internationalem Weg gegen hohe Kosten zu beziehen, sodass sich auch diese Alternative der klinischen Anwendung nicht eröffnet hat.

Auch wenn es in der Literatur Mitteilungen über das Überleben mit extrem niedrigen Hämoglobinwerten, gerade auch bei Glaubensangehörigen der Zeugen Jehovas, gibt $[27,28,29,30,31]$, muss an dieser Stelle festgestellt werden, dass bei Vorhandensein von Komorbiditäten wie arteriellen Stenosen, koronarer Herzkrankheit, Herzinsuffizienz, „chronic obstructive pulmonary disease" (COPD) u.v.a. bereits bei deutlich höheren Hämoglobinkonzentrationen lebensgefährliche Komplikationen und letztlich - auch ohne Begleiterkrankungen - der Tod des Patienten eintreten kann [32]. Es ist daher erforderlich, z. B. vor Anwendung einer kontrollierten Hypotension, kardiozirkulatorische Risikofaktoren auszuschließen. Weiterhin sind ein invasives Monitoring und eine engmaschige Überwachung der Oxygenierungs- sowie Herzkreislaufparameter essenzielle Voraussetzungen für eine extreme Hämodilution. Nicht unerwähnt bleiben kann in diesem Zusammenhang, dass durch den Einsatz von Präparaten wie Erythropoetin, durch invasives Monitoring und Überwachung auf der Intensivstation über viele Tage aufgrund einer permissiven Anämie - im Vergleich zur Transfusion einer überschaubaren Anzahl von Erythrozytenkonzentraten - hohe Kosten auf die behandelnden Abteilungen zukommen. Dies ist im Zeitalter der Kostenpauschalen durch die Diagnosis Related Groups (DRGs) - und im Zusammen- hang mit den möglicherweise durch Unterlassung oder Durchführung einer vom Patienten nichtgewollten Transfusion von Blut und Blutprodukten hervorgerufenen forensischen sowie für den durchführenden Anästhesisten schwierigen Gewissensentscheidung - kritisch anzumerken.

\section{จ Die perioperative Behandlung von Zeugen Jehovas bereitet bei guter Vorbereitung wenige Probleme}

Auf der anderen Seite kann konstatiert werden, dass bei Auseinandersetzung mit dieser Problematik und Annahme der medizinischen Herausforderung die perioperative Behandlung von Zeugen Jehovas bei guter Vorbereitung, insbesondere bei elektiven Eingriffen, in der Regel wenige Probleme bereitet. Der Beitrag trägt in seiner umfassenden Darstellung möglicher transfusionsvermeidender Behandlungsalternativen zur Sicherheit des perioperativen Managements der Patienten aus der Glaubensgemeinschaft der Zeugen Jehovas bei, kann aber selbstverständlich die Einzelfallentscheidung der agierenden Personen nicht ersetzen.

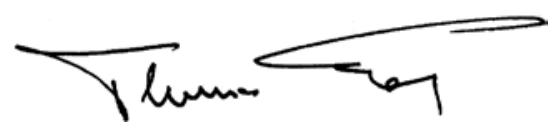

T. Standl

\section{Korrespondenzadresse}

\section{Prof. Dr. T. Standl}

Klinik für Anästhesie, Operative Intensivund Palliativmedizin, Städtisches Klinikum Solingen, Akademisches Lehrkrankenhaus der Universität Köln Gotenstraße 1, 42653 Solingen standl@klinikumsolingen.de

\section{Literatur}

1. Biermann E (1993) Forensische Gesichtspunkte der Bluttransfusion. Anaesthesist 42:187-202

2. Ulsenheimer K (2010) Ablehnung von Fremdblut durch Zeugen Jehovas. Perioperatives Management aus rechtlicher Sicht. Anaesthesist 59:312318

3. OLG München (2003) Urt v. 31.01.2002 1 U 4705/98. MedR 3:174

4. Bock RW (1996) Juristischer Kommentar zur Ablehnung von Bluttransfusionen. Anasthesiol Intensivmed Notfallmed Schmerzther 31:506-507

5. Baur U (2006) Bluttransfusion bei Zeugen Jehovas. Gynakologie 39:920-921
6. Röttgers HR, Nedjat S (2002) Kritik am Transfusionsverbot nimmt zu. Dtsch Arztebl 99:A102-105

7. Greenburg AG (1995) A physiologic basis for red blood cell transfusion decisions. Am J Surg 170:44S-48S

8. Habler O, Voß B (2010) Perioperatives Management bei Zeugen Jehovas. Spezielle Berücksichtigung der religiös motivierten Ablehnung von Fremdblut. Anaesthesist 59:297-311

9. Adam G (2001) Interventionelle radiologische Maßnahmen. Anasthesiol Intensivmed Notfallmed Schmerzther 36 [Suppl 2]:130-133

10. Jacob M, Chapell D, Hofmann-Kiefer K et al (2007) Determinanten des insensiblen Flüssigkeitsverlustes. Perspiratio, Proteinshift und endotheliale Glykokalyx. Anaesthesist 56:747-64

11. Boldt J, Weber A, Mailer Ket al (1999) Acute normovolaemic haemodilution vs controlled hypotension for reducing the use of allogeneic blood in patients undergoing radical prostatectomy. $\mathrm{Br} \mathrm{J}$ Anaesth 82:170-174

12. Standl T (2000) Artificial oxygen carriers as red blood cell substitutes: perfluorocarbons and cellfree hemoglobin. Infusionsther Transfusionsmed 27:128-137

13. Spahn DA, Waschke K, StandI T et al (2002) Use of perflubron emulsion to decrease allogeneic blood transfusion in high-blood-loss non-cardiac surgery: results of a European phase 3 study. Anesthesiology 97:1338-1349

14. StandIT (2001) Hemoglobin-based erythrocyte transfusion substitutes. Expert Opin Biol Ther 1:831-843

15. StandIT (2004) Artificial oxygen carriers: haemoglobin-based oxygen carriers (HBOC) - Current status 2004. Transfus Med Hemother 31:262-268

16. LaMuraglia GM, O'Hara PJ, Baker WH et al (2000) The reduction of the allogeneic transfusion requirement in aortic surgery with a hemoglobinbased solution. J Vasc Surg 31:299-308

17. Standl T Burmeister MA, Horn EP et al (1998) Bovine haemoglobin-based oxygen carrier for patients undergoing haemodilution before liver resection. Br J Anaesth 80:189-194

18. Mullon J, Giacoppe G, Clagett C et al (2000) Transfusions of polymerized bovine hemoglobin in a patient with severe autoimmune hemolytic anemia. N Engl J Med 342:1638-1643

19. Anton N, Hitzler JK, Kavanagh BP (2002) Treatment of life-threatening post-haemorrhagic anaemia with cell-free haemoglobin solution in an adolescent Jehovah's Witness. Br J Haematol 118:11831186

20. Cothren CC, Moore EE, Long JS et al (2004) Large volume polymerized haemoglobin solution in a Jehovah's Witness following abruptio placentae. Transfus Med 14:241-246

21. Lanzinger MJ, Niklason LE, Shannon M, Hill SE (2005) Use of hemoglobin raffimer for postoperative life-threatening anemia in a Jehovah's Witness. Can J Anaesth 52:369-373

22. Niquille M, Touzet M, Leblanc I, Baron JF (2000) Reversal of intraoperative myocardial ischemia with a hemoglobin-based oxygen carrier. Anesthesiology 92:882-885

23. Burmeister MA, Rempf C, Standl T et al (2005) Effects of prophylactic or therapeutic application of bovine haemoglobin HBOC-200 on ischaemiareperfusion injury following acute coronary ligature in rats. Br J Anaesth 95:737-45 
24. Freitag M, Standl TG, Kleinhans H et al (2006) Improvement of impaired microcirculation and tissue oxygenation by hemodilution with hydroxyethyl starch plus cell-free hemoglobin in acute porcine pancreatitis. Pancreatology 6: 232-239

25. Rempf C, StandI T, Gottschalk A et al (2008) Effects of a prophylactic or therapeutic application of perflubron emulsion on myocardial ischaemia-reperfusion injury in rats. Eur J Anaesthesiol 25:850-859

26. Rempf C, StandI T, Schenke K et al (2009) Administration of bovine polymerized haemoglobin before and during coronary occlusion reduces infarct size in rabbits. Br J Anaesth 103:496-504

27. Busse J, Wesseling C (1996) Tolerierung eines extremen intraoperativen Blutverlustes bei einer Zeugin Jehovas. Anasthesiol Intensivmed Notfallmed Schmerzther 31:498-501

28. Teßmann R, Lüpke U von (1996) Überleben einer schwersten Blutungsanämie bei einer Zeugin Jehovas. Anasthesiol Intensivmed Notfallmed Schmerzther 31:501-504

29. Howell PJ, Bamber PA (1987) Severe acute anaemia in a Jehovah's Witness. Survival without blood transfusion. Anaesthesia 42:44-48

30. Brimacombe J, Skippen P, Talbutt P (1991) Acute anaemia to a haemoglobin of $14 \mathrm{~g} / \mathrm{l}$ with survival. Anaesth Intensive Care 19:581-583

31. Boettcher W, Merkle F, Huebler M et al (2005) Transfusion-free cardiopulmonary bypass in Jehovah's Witness patients weighing less than 5 kg. J Extra Corpor Technol 37:282-285

32. Schweitzer M, Oswald PM (1996) Letaler hämorrhagischer Schock bei einem Zeugen Jehovas. Anasthesiol Intensivmed Notfallmed Schmerzther 31:504-506

\section{E. Ullbricht \\ Wenn Patienten nicht zahlen}

Heidelberg: Springer 2009, 242 S., (ISBN 978-3-540-79481-3), 39.95 EUR

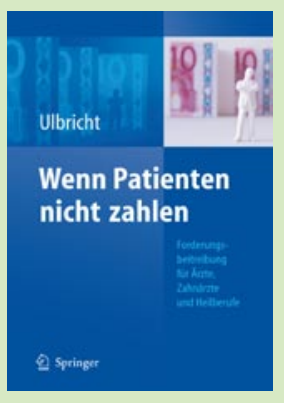

Das Buch „Wenn

Patienten nicht

zahlen" aus der

Feder einer Juristin und Unternehmensberaterin befasst sich mit der Forderungsbeitreibung für Ärzte, Zahnärzte und Heilberufe.

Im Vorwort wird postuliert, Ärzte müssten sich immer häufiger um andere Einnahmequellen bemühen, die Einnahmen aus den Kassenarztverträgen würden „bei Weitem“ nicht ausreichen.,Jetzt" seien auch unternehmerische Fähigkeiten gefragt und Hilfe soll dieses Buch bieten. Es handelt vom Weg des Arztes zum Unternehmer (Kapitel A), beschreibt unternehmerische Strategien und ärztliche Rechts- und Standespflichten (Kapitel B) sowie ein erfolgreiches Forderungsmanagement (Kapitel C) - z. B. Bonitätsprüfung, Behandlungsvertrag, Honorarvereinbarung, Mahnung, Insolvenz des Patienten. Es folgt die gerichtliche Geltendmachung (Kapitel D), Schuldnertricks (Kapitel E), und das Outsourcing der Forderungsbeitreibung wird als Lösung diskutiert (Kapitel F). Im Text wird der Leser häufig direkt angesprochen: „Sie als Arzt ", ,Ihr Unternehmen“, ,, was Sie ankündigen...., dies innerhalb eines Textes mit Ausführungen, die juristische Laien jedenfalls teilweise nicht mehr inhaltlich erfassen können. Verlässliche Zahlen zum Ausmaß der Zahlungsverweigerung durch Patienten werden nicht präsentiert, es wird jedoch darauf hingewiesen, dass eine Arztpraxis nach Angaben einer führenden ärztlichen Verrechnungsstelle im Durchschnitt „nur" ca. $3 \%$ ihres Umsatzes abschreiben müsse.

Dennoch: Das ohne Spalten und schwarz-weiß gedruckte Buch mit grau unterlegten Hervorhebungen und Auflistungen ist engagiert geschrieben, juristisch fundiert und gibt dem Arzt eine Vorstellung davon, wie schwierig und kompli- ziert es sein kann, einen (ehemaligen) Patienten rechtlich korrekt zur Zahlung zu bewegen. Die Ärztin bzw. der Arzt, obwohl angesprochen als Zielgruppe, können das Buch wohl eher zur Orientierung bzw. zum Nachschlagen verwenden, und selbst dann darf häufig bezweifelt werden, dass sie/er allein dadurch wirkliche Hilfe bekommt. Ärzten wird vor allem vermittelt, dass sie - wenn Patienten nicht zahlen sich an Profis wie die Autorin wenden sollten, und für diese professionelle Zielgruppe ist das Buch sicher hilfreich. Es ist, so gesehen, teilweise auch Werbung in eigener Sache.

R. Dettmeyer (Gießen) 